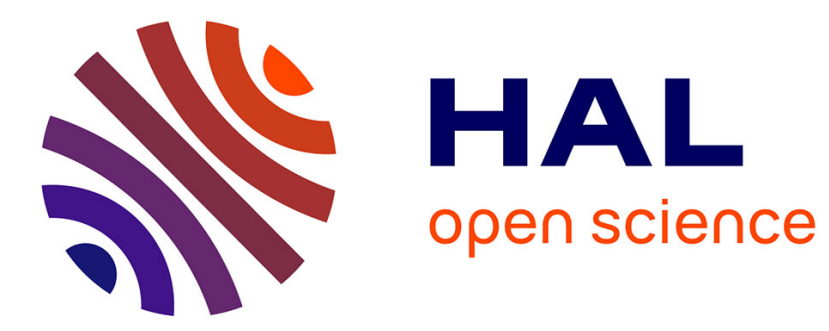

\title{
NONLINEAR WAVES IN HOMOGENEOUS AND HETEROGENEOUS ELASTIC SOLIDS
}

\author{
A. Kluwick, A. Nayfeh
}

\section{To cite this version:}

A. Kluwick, A. Nayfeh. NONLINEAR WAVES IN HOMOGENEOUS AND HETEROGENEOUS ELASTIC SOLIDS. Journal de Physique Colloques, 1979, 40 (C8), pp.C8-207-C8-211. 10.1051/jphyscol:1979835 . jpa-00219540

\section{HAL Id: jpa-00219540 https://hal.science/jpa-00219540}

Submitted on 1 Jan 1979

HAL is a multi-disciplinary open access archive for the deposit and dissemination of scientific research documents, whether they are published or not. The documents may come from teaching and research institutions in France or abroad, or from public or private research centers.
L'archive ouverte pluridisciplinaire HAL, est destinée au dépôt et à la diffusion de documents scientifiques de niveau recherche, publiés ou non, émanant des établissements d'enseignement et de recherche français ou étrangers, des laboratoires publics ou privés. 


\title{
NONLINEAR WAVES IN HOMOGENEOUS AND HETEROGENEOUS ELASTIC SOLIDS
}

\author{
A. Kluwick and A.H. Nayfeh
}

Institut für Strömungslehre Technische Universitat Wien, Karlsplatz 13, A 1040, Vienna, Austria. Department of Engineering Science and Mechanics Virginia Polytechnic Institute and State University Blacksburg, Virginia 24061.

Résumé.- La mêthode des échelles multiples est utilisēe pour déterminer des développements uniformes au second ordre de déplacements qui décrivent des ondes planes non linéaires se propageant dans un demi-espace élastique. Les propriétés du matériau peuvent être homogènes ou varier lentement selon la direction de propagation. L'interaction entre les ondes de dilatation et de cisaillement ainsi que 1 'interaction entre propriétés non linéaires et hétērogénéité du milieu sont discutées. Les rẻsultats et les méthodes de rēsolution proposées sont comparës aux rēsultats antérieurs obtenus en utilisant la méthode analytique dite des caractêristiques.

Abstract. - The method of multiple scales is used to determine second-order uniform expansions for the displacements describing nonlinear plane waves propagating into an elastic half space. The material properties may be homogeneous or they may vary slowly in the direction of propagation. A discussion is presented for the interaction between dilatational and shear waves as well as the interaction between the nonlinearity and the heterogeneity. The present results and method of solution are compared with those obtained earlier for homogeneous and slightly heterogeneous media by using the analytic method of characteristics.

\section{Introduction. - The method of multiple scales} $11 /$ is used to analyse the propagation of finiteamplitude longitudinal and shear waves in a hal, $f$ space whose material properties vary slowly with position. The material constitutive relations are assumed to be elastic but nonlinear. For a comprehensive review of nonlinear propagation in heterogeneous materials, we refer the reader to Nayfeh and Mook /2/.

The problem of finite-amplitude longitudinal and shear waves propagating in a homogeneous isotropic half space was studied by Davison $/ 3 /$. He obtained a second-order uniform expansion by using the analytic method of characteristics /4,1/. Nayfeh / $1 /$ extended Davison's work to the case of anisotropic materials, while Nair and Nemat-Nasser /5/ extended Davison's work by including the effect of a sma 11 heterogeneity.

The purpose of the present paper is to analyze the propagation of finite-amplitude longitudinal and shear waves in a heterogeneous half space, whose material properties vary slowly with position but the heterogeneity need not be small. The analysis is carried out by using the method of multiple scales rather than the analytic method of characteristics ; it is not clear yet how the latter method can be applied to this problem.

2. Problem Formulation.- We consider the propagation of plane longitudinal and shear waves in heterogeneous, nonlinear elastic solids. We introduce dimensionless quantities by using a reference length $x_{r}$, a reference wave speed $c_{r}$ and a reference density $\rho_{r}$. If $(x, y)$ and $(x, y)$ denote the positions of a particle in the undeformed and feformed states, respectively, and $(u, v)$ denote the displacements of this particle, then

$$
x=-x+u(x, t) \text { and } Y=y+v(x, t)
$$

where the deformation is assumed to depend on $x$ only. The equations governing the deformation are statements of the conservation of mass and linear momentum ; that is,

$\frac{\rho 0}{\rho}=\frac{\partial(X, Y)}{\partial(X, Y)}=1+\frac{\partial u}{\partial X}$

$$
\rho^{0} \frac{\partial^{2} u}{\partial t^{2}}=\frac{\partial \sigma}{\partial x} \text { and } \frac{\partial^{2} v}{\partial t^{2}}=\frac{\partial \tau}{\partial x}
$$

where $\rho_{0}$ is the density of the medium in the undeformed state and $\sigma$ and $\tau$ are the longitudinal and shear stresses. In what follows, we assume that they are analytic functions of the strains $u_{x}$ and $v_{x}$ so that they can be expanded in Tayior series as

$\sigma=\rho_{0}\left(c_{p}^{2} u_{x}+\frac{1}{2} a_{1} u_{x}^{2}+a_{2} u_{x} v_{x}+\frac{1}{2} a_{3} v_{x}^{2}+\ldots\right)$ 
$\left.\tau=\rho_{0} f_{s}^{2} v_{x}+\frac{1}{2} b_{1} u_{x}^{2}+b_{2} u_{x} v_{x}+\frac{1}{2} b_{3} v_{x}^{3}+\ldots\right)$

where $c_{p}$ and $c_{s}$ are the linear longitudinal and shear wave speeds. In what follows, we assume that the properties of the medium vary slowly with $x$ so that we can consider $\rho_{0}, c_{p}, c_{s}, a_{j}$ and $b_{j}$ to be functions of a long scale $x_{1}=\varepsilon x$, where $\varepsilon$ is a small but finite dimensionless quantity.

To complete the problem formulation, we need to specify the initial and boundary conditions. In this study, we consider the so-called signalling problem; that is,

$P(x, 0)=Q(x, 0)=R(x, 0)=S(x, 0)=0$ for $x>0$

$P(0, t)=\varepsilon \phi(t), Q(0, t)=\varepsilon \psi(t)$ for $t>0$

where

$P=u_{x}, \quad Q=v_{x}, \quad R=u_{t}, \quad S=v_{t}$

so that

$R_{x}-P_{t}=0, \quad S_{x}-Q_{t}=0$

3. Approximate Solution.- We note that the initial conditions are taken to be small to enable us to determine a solution for small but finite amplitude waves. Following the method of multiple scales $/ 1 /$, we let

$P=\varepsilon P_{1}\left(s_{1}, s_{2}, x_{1}\right)+\varepsilon^{2} P_{2}\left(s_{1}, s_{2}, x_{1}\right)+\ldots$

$Q=\varepsilon Q_{1}\left(s_{1}, s_{2}, x_{1}\right)+\varepsilon^{2} Q_{2}\left(s_{1}, s_{2}, x_{1}\right)+\ldots$

$R=\varepsilon R_{1}\left(s_{1}, s_{2}, x_{1}\right)+\varepsilon^{2} R_{2}\left(s_{1}, s_{2}, x_{1}\right)+\ldots$

$S=\varepsilon S_{1}\left(s_{1}, s_{2}, x_{1}\right)+\varepsilon^{2} S_{2}\left(s_{1}, s_{2}, x_{1}\right)+\ldots$

where

$s_{1}=t-\int \frac{d x}{c_{p}} \quad, s_{2}=t-\int \frac{d x}{c_{s}}$.

are the linear characteristics for right-running waves.
Substituting equations (9) - (13) into equations (3) $-(5)$ and (8), using equation (7), and equating the coefficients of $\varepsilon$ on both sides, we obtain

$\frac{\partial R_{1}}{\partial S_{1}}+\frac{\partial R_{1}}{\partial s_{2}}+c_{p}^{2}\left[\frac{1}{c_{p}} \frac{\partial P_{1}}{\partial S_{1}}+\frac{1}{c_{s}} \frac{\partial P_{1}}{\partial s_{2}}\right]=L_{1}\left(P_{I}, R_{1}\right)=0$

$\frac{1}{c_{p}} \frac{\partial R_{1}}{\partial S_{1}}+\frac{1}{c_{S}} \frac{\partial R_{1}}{\partial S_{2}}+\frac{\partial P_{1}}{\partial S_{1}}+\frac{\partial P_{1}}{\partial S_{2}}=L_{2}\left(P_{1}, R_{1}\right)=0$

$\frac{\partial S_{1}}{\partial S_{1}}+\frac{\partial S_{1}}{\partial S_{2}}+c_{S}^{2}\left[\frac{1}{c_{p}} \quad \frac{\partial Q_{1}}{\partial S_{1}}+\frac{1}{c_{S}} \frac{\partial Q_{1}}{\partial S_{2}}\right]=L_{3}\left(Q_{1}, S_{1}\right)=0$

$L_{2}\left(Q_{1}, S_{1}\right)=0$

Since in the initial state the medium was at rest and since it is assumed to be semi-infinite, the solution of the first-order problem consists of right-running waves only; that is,

$$
\begin{array}{ll}
P_{1}=f\left(s_{1}, x_{1}\right), & R_{1}=-c_{p} f\left(s_{1}, x_{1}\right) \\
Q_{1}=g\left(s_{2}, x_{1}\right) & s_{1}=-c_{s} g\left(s_{2}, x_{1}\right)
\end{array}
$$

Substituting equations (9) - (13) into equations (3) - (5) and (8), using equations (7) and (18), and equating the coefficients of $\varepsilon^{2}$ on both sides, we obtain

$$
\begin{aligned}
& L_{1}\left(P_{2}, R_{2}\right)=-c_{p} \frac{\partial}{\partial x_{1}}\left(c_{p} f\right)-a_{\bar{z}}\left[\frac{1}{c_{p}} \frac{\partial}{\partial s_{1}}\right. \\
& \left.+\frac{1}{c_{s}} \frac{\partial}{\partial s_{2}}\right](f g)-\frac{a_{3}}{c_{s}} g \frac{\partial g}{\partial s_{2}}+H\left(s_{1}, x_{1}\right)
\end{aligned}
$$

$$
\begin{aligned}
& L_{2}\left(P_{2}, R_{2}\right)=-\frac{\partial}{\partial x_{1}}\left(c_{p} f\right) \\
& L_{3}\left(Q_{2}, S_{2}\right)=-c_{s} \frac{\partial}{\partial x_{1}}\left(c_{s} g\right)-b_{2}\left[\frac{1}{c_{p}} \frac{\partial}{\partial s_{1}}\right.
\end{aligned}
$$


$\left.+\frac{1}{c_{s}} \frac{\partial}{\partial s_{2}}(f g)\right]-\frac{b_{1}}{c_{p}} \quad \frac{\partial}{\partial s_{1}}+J\left(s_{2}, x_{1}\right)$

$L_{2}\left(Q_{2}, S_{2}\right)=-\frac{\partial}{\partial x_{1}}\left(c_{s} g\right)$

where

$$
\begin{aligned}
& H\left(s_{1}, x_{1}\right)=2 c_{p}^{2} \frac{\partial f}{\partial x_{1}}+c_{p}^{2} f \frac{d}{d x_{1}}\left[\ln \left(\rho_{0} c_{p}^{3}\right)\right] \\
& -\frac{a_{1}}{c_{p}} f \frac{\partial f}{\partial s_{1}} \\
& J\left(s_{2}, x_{1}\right)=2 c_{s}^{2} \frac{\partial g}{\partial x_{1}}+c_{s}^{2} g \frac{d}{d x_{1}}\left[\ln \left(\rho_{0} c_{s}^{3}\right)\right] \\
& -\frac{b_{3}}{c_{s}} g \frac{\partial g}{\partial s_{2}}
\end{aligned}
$$

The second and third terms on the right-hand side of equation (19) account for the effect of the shear wave on the longitudina? wave, while the remaining terms account for the effect of the longitudinal wave on itself. Similarly, the second and third terms on the right-hand side of equation (21) account for the effect of the longitudinal wave on the shear wave, while the remaining terms account for the effect of the shear wave on itself.

A particular solution of equations (19) and (20) is

$$
P_{2 p}=\frac{c_{s}}{c_{p}\left(c_{p}-c_{s}\right)} s_{2} H\left(s_{1}, x_{1}\right)-\frac{a_{3}}{2\left(c_{p}^{2}-c_{s}^{2}\right)} g^{2}
$$

$+F_{1}\left(s_{1}, s_{2}, x_{1}\right)$

$R_{2 p}=-\frac{c_{s}}{\left(c_{p}-c_{s}\right)} s_{2} H\left(s_{1}, x_{1}\right)-c_{p} \int^{s_{1}} \frac{\partial}{\partial x_{1}}\left(c_{p} f\right) d s_{1}$

$$
+\frac{a_{3} c_{s}}{2\left(c_{p}^{2}-c_{s}^{2}\right)} g^{2}+G_{1}\left(s_{1}, s_{2}, x_{1}\right)
$$

where

$$
\begin{aligned}
& G_{1}=-\frac{a_{2} c_{s}}{2\left(c_{p}-c_{s}\right)} \iint_{A_{\ell}}\left[\frac{1}{c_{p}^{2}} \frac{\partial^{2}}{\partial s_{1}^{2}}+\frac{2}{c_{p} c_{s}} \frac{\partial^{2}}{\partial s_{1} \partial s_{2}}\right. \\
& +\frac{1}{c_{s}^{2}} \frac{\left.\partial^{2}\right](f g) d s_{1} d s_{2}}{\partial s_{2}^{2}}
\end{aligned}
$$

$G_{1}=\frac{a_{2} c_{s}}{2\left(c_{p}-c_{s}\right)} \iint\left[\frac{1}{c_{p}} \frac{\partial^{2}}{\partial s_{1}^{2}}+\left(\frac{1}{c_{p}}+\frac{1}{c_{s}}\right) \frac{\partial^{2}}{\partial s_{1} \hat{\partial} s_{2}}\right.$ $A_{\ell}$

$\left.+\frac{1}{c_{s}} \frac{\partial^{2}}{\partial s_{2}^{2}}\right](f g) d s_{1} d s_{2}$

and $A_{l}$ is the domain shown in Fig.1. In equations (25) and (26), there are cumulative (secular) terms which make $\varepsilon P_{2}$ and $\varepsilon R_{2}$ larger than the order of $\varepsilon P_{1}$ and $\varepsilon R_{1}$ for all $x>0\left(\frac{1}{\varepsilon}\right)$ or $t>0\left(\frac{1}{\varepsilon}\right)$. If $\phi(t)$ and $\psi(t)$ are periodic or if they are pulses, then $F_{1}$ and $G_{1}$ are bounded and hence not cumulative. In this case, the only cumulative terms are the ones proportional to $s_{2} H\left(s_{1}, x_{1}\right)$. Hence for a uniform expansion, $H\left(s_{1}, x_{1}\right)=0$. Following

Nayfeh $/ 6 /$, we find that the solution of $H\left(s_{1}, x_{1}\right)$ $=0$ is

$P=-\frac{1}{c_{p}} R=\varepsilon\left[\frac{\rho_{0}(0) c_{p}(0)}{\rho_{0} c_{p}^{3}}\right]^{1 / 2} \cdot \phi(\xi)+\ldots$

where

$$
\left.\xi=s+\frac{1}{2} \phi(\xi) \int_{0}^{x} \frac{a_{1}}{c_{p}^{3}} \underset{p}{\left[\rho_{0} c^{3}\right.}{ }_{p}(0) c_{p}^{3}(0)\right]^{1 / 2} d x_{1}+\ldots
$$

Thus, to first order the shear wave does not affect the longitudinal wave. However, equations (25) and (26) show that the shear wave does affect the longitudinal wave at second order.

The analysis of equations (21) and (22) is analogous to equations (19) and (20). Again the wave-interaction terms do not give rise to cumula- 


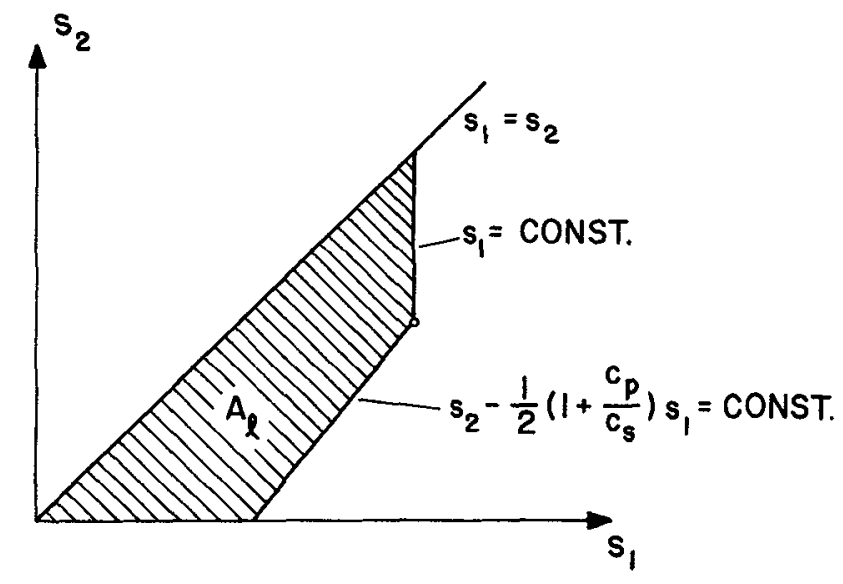

FIG.1 : Domain of integration for longitudinal wave.

tive terms effects and the elimination of cumulative terms leads to $J\left(s_{1}, x_{1}\right)=0$. Hence

$Q=-\frac{1}{c_{s}} S_{1}=\varepsilon\left[\frac{\rho_{0}(0) c_{s}^{3}(0)}{\rho_{0} c_{s}^{3}}\right]^{1 / 2} \psi(n)+\ldots$

where

$n=s_{1}+\frac{1}{2} \psi(\eta) \int_{0}^{x_{1}} \frac{b_{3}}{2 c_{s}^{3}} \frac{\left.\rho_{0}(0) c_{s}(0)\right]^{1 / 2} c_{s}^{3}}{\rho_{0}} d x_{1}+\ldots$

Moreover,

$Q_{2}=\frac{b_{1} f^{2}}{2\left(c_{p}^{2}-c_{s}^{2}\right)}+F_{2}$

$s_{2 p}=-c_{s} \int_{0}^{s_{z}} \frac{\partial}{\partial x_{1}}\left(c_{s} g\right) d s_{2}-\frac{b_{1} c_{p}}{2\left(c_{p}^{2}-c_{s}^{2}\right)} f^{2}+G_{2}$

where

$F_{2}=\frac{c_{p} b_{2}}{2\left(c_{p}-c_{s}\right)} \iint_{A_{s}}\left[\frac{1}{c_{p}^{2}} \frac{\partial^{2}}{\partial s_{1}^{2}}+\frac{2}{c_{p} c_{s}} \frac{\partial^{2}}{\partial s_{1} \partial s_{2}}\right]$
$+\frac{1}{c_{s}^{2}} \frac{\partial^{2}}{\partial s_{2}^{2}}(f g) d s_{1} d s_{2}$

$$
G_{2}=\frac{c_{p} b_{2}}{2\left(c_{p}-c_{s}\right)} \iiint_{A_{S}} \frac{1}{c_{p}} \frac{\partial^{2}}{\partial s_{1}^{2}}+\left(\frac{1}{c_{p}}+\frac{1}{c_{s}}\right) .
$$

$$
\left.\frac{\partial^{2}}{\partial s_{2}^{2}}+\frac{1}{c_{s}^{2}} \frac{\partial^{2}}{\partial s_{2}^{2}}\right](f g) d s_{1} d s_{2}
$$

The domain $A_{S}$ is shown in Fig. 2. Again, if $\phi(t)$ and $\psi(t)$ are periodic or pulses, the integrals in equations (33) and (34) are bounded and hence not cumulative. Therefore, the expansion (31) and (32) is uniform and the shear wave is not affected by the longitudinal wave to first order.

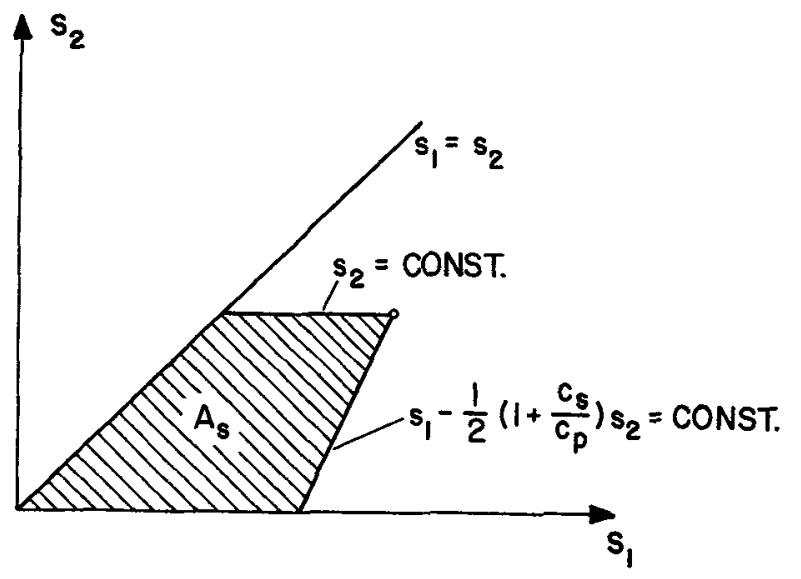

FIG. 2 : Domain of integration for shear wave.

4. Shock Waves.- The results of the preceding section show that to first order there is no interaction between longitudinal and shear waves. Hence, it is sufficient to analyze one of them, say longitudinal waves.

Equation (29) and (30) show that the speed of a longitudinal wave depends on its local amplitude. Thus, at some distance $x$, the solution (29) and (30) becomes multi-valued due to the steepening of the wave-form. Since neither the stress nor the strain can be muti-valued in space or time, a shock wave forms. The shock formation distance is the smallest distance at which the field variables exhibit infinite slopes in either space or time. Differentiation of equation (29) with respect to $x$ shows that $\partial P / \partial x$ and $\partial R / \partial x$ firs't become infinite, and hence a shock develops, at a distance $x$ given by

$$
1-\frac{1}{2} \phi^{\prime}\left(\xi_{s}\right) \int_{0}^{x} \frac{a_{1}}{c_{p}^{3}}\left[\frac{\rho_{0}(0) c_{p}^{3}(0)}{\rho^{0} c_{p}^{3}}\right]^{1 / 2} d x_{1}=0
$$


where $\xi=\xi_{S}$ is the characteristic for which $\varepsilon a_{1} \phi^{\prime}$ $\left(\xi_{s}\right)>0$ and $\left|\phi^{\prime}(\xi)\right|$ attains its maximum. Similar$1 y$, a shear shock develops at an $x$ given by

$1-\frac{1}{2} \psi^{\prime}\left(\eta_{s}\right) \int_{0}^{x_{2}} \frac{b_{1}}{c_{s}^{3}} \frac{\rho_{0}(0) c^{3}(0) 1 / 2}{\rho_{0} c_{s}^{3}} d x_{1}=0$

where $n=n_{s}$ is the characteristic for which $\varepsilon b_{1}$ $\psi^{\prime}\left(n_{s}\right)>0$ and $\left|\Psi^{\prime}(n)\right|$ attains its maximum.

Shock waves can also be located by determining the deformations $u$ and $v$ from $P$ and $Q$. Then the shoks can be located by following Nayfeh and Kluwick /7/ and imposing the condition that the displacements are continuous across the shocks.

5. Comparison with the Method of Characteristics.The propagation of finite-amplitude waves in homogeneous elastic solids was studied by Davison /3/ by using the analytic method of characteristics /4,1/. Davison's results were extended to solids with a more general constitutive equation by Nayfeh /1/ and to heterogeneous solids by Nair and Nemat-Nasser $/ 5 /$.

Using the analytic method of characteristics, one expands both the dependent and independent variables in terms of the exact outgoing characteristics. In the case of homogeneous solid, one obtains /1/

$$
\begin{aligned}
& P=\varepsilon \phi(\xi)+\ldots, \quad R=-\varepsilon C_{p} \phi(\xi)+\ldots \\
& Q=\varepsilon \psi(n)+\ldots, \quad S=-\varepsilon c_{S} \psi(n)+\ldots
\end{aligned}
$$

where

$$
\begin{aligned}
& t=-\frac{x}{c_{p}}=\xi-\frac{\varepsilon c_{s}}{2 c_{p}\left(c_{p}^{2}-c_{s}\right)}\left[a_{1}(\xi-n) \phi(\xi)\right. \\
& \left.-a_{2} \int_{\xi}^{\eta} \psi(\tau) d \tau\right]+\ldots \ldots \\
& t-\frac{x}{c_{s}}=\eta-\frac{\varepsilon c_{p}}{2 c_{s}^{2}\left(c_{p}-c_{s}\right)}\left[b_{3}(\eta-\xi) \psi(\eta)\right. \\
& \left.-b_{2} \int_{n} \phi(\tau) d \tau\right]+\ldots .
\end{aligned}
$$

We note that the last term: on the right-hand sides of equations (40) and (41) coscribe a nopli- near interaction between the longitudinal and shear waves. Other interaction terms are present in the second-order quantities /3/. If the integrals of $\phi$ and $\psi$ are bounded, then the interaction terms are small compared with the remaining terms in equations (40) and (41) which are unbounded with either $x$ or $t$. Hence, one can expand equations (39) in Taylor series by assuming the terms proportional to $a_{2}$ and $b_{2}$ to be sma11. The result is the same as the one obtained from our solution in the case of of a homogeneous medium. If the integrais of $\phi$ and $\psi$ are unbounded, one can include these terms in the condition for the elimination of secular terms and hence obtain a solution in agreement with equations (39) - (41). Alternatively, one can inspect the expressions for $P_{2}, R_{2}, Q_{2}$ and $S_{2}$, use the method of renormalization /1/ in conjunction with the already obtained solution of Sect. 3 , and obtain an expansion that reduces to (39) - (41) for a homogeneous solid.

In the case of the weakly heterogeneous medium studied by Nair and Nemat-Nasser /5/, we have

$c_{p}=c_{p_{0}}\left[1+\varepsilon c_{p_{1}}(x)\right], \quad c_{s}=c_{s_{0}}\left[1+\varepsilon c_{s_{1}}(x)\right]$

Substituting these expressions into equations (29) (32) shows that the resulting expansion is equivalent to that of Nair and Nemat-Nasser when the interaction terms are negligible. If they are not, our results can be modified as discussed above to obtain an exparision that is the same as that of Nair and Nemat-Nasser. However, whereas the method of multiple scales can treat systems with slowly varying properties which need not be small, the analytic method of characteristics has not been applied yet to cases of large heterogeneities.

\section{References}

/1/ Nayfeh, A.H., Perturbation Methods (Wiley Interscience, New York, 1973).

12) Nayfeh, A.H. and Mook, D.T., Nonlinear Oscillations (Wiley Interscience, New York, 1979).

13/ Davison, L., Int. J. Solids Struct. 4 (1968) 301.

14/ Lin, C.C., J. Math. Phys. 33 (1954) 117.

15/ Nair, S. and Nemat-Nasser, S., Int. J. Eng. Sci. 9 (1971) 1087.

16/ Nayfeh, A.H., J. Sound Vib. 42 (1975) 357.

17/ Nayfeh, A.H. and Kluwick, A., J. Sound Vib. 48 (1976) 293. 